\title{
Teaching Integer Security Using Simple Visualizations
}

\author{
James Walker \\ Man Wang \\ jwwalker@mtu.edu \\ manw@mtu.edu \\ Michigan Technological University \\ Houghton, MI
}

\author{
Steven Carr \\ Western Michigan University \\ Kalamazoo, MI \\ steve.carr@wmich.edu
}

\author{
Jean Mayo \\ Ching-Kuang Shene \\ jmayo@mtu.edu \\ shene@mtu.edu \\ Michigan Technological University \\ Houghton, MI
}

\begin{abstract}
Integer errors can introduce significant vulnerabilities into $\mathrm{C}$ programs. We have developed a program analysis and visualization tool to help students understand integer representation and type conversions with the goal to help students avoid introducing these errors into the code they develop. The visualization is through the Integer Representation (IR) window within a larger system for analysis and visualization of security issues in $\mathrm{C}$ programs. The system is called the Visualization and Analysis for C Code Security (VACCS) system. In this paper, we describe our experience with teaching fundamental aspects of integer security in a junior-level systems programming course, the IR window, and an evaluation of the tool. Our results indicate that students found the tool to be useful and that it enhanced the course in which it was used.
\end{abstract}

\section{CCS CONCEPTS}

- Social and professional topics $\rightarrow$ Computing education.

\section{KEYWORDS}

Integer security; visualization; cybersecurity education

\section{ACM Reference Format:}

James Walker, Man Wang, Steven Carr, Jean Mayo, and Ching-Kuang Shene. 2019. Teaching Integer Security Using Simple Visualizations. In Innovation and Technology in Computer Science Education (ITiCSE '19), July 15-17, 2019, Aberdeen, Scotland UK. ACM, New York, NY, USA, 7 pages. https: //doi.org/10.1145/3304221.3319760

\section{INTRODUCTION}

Naive and sloppy use of integers within a C program can introduce subtle but serious security vulnerabilities. This is especially dangerous because many systems-level programs are written in $\mathrm{C}$ for performance reasons and these systems-level programs are fundamental to protection of information.

While undergraduate courses in computer organization and $\mathrm{C}$ programming commonly cover integer representation and type conversions, we have found that even after taking these courses, many students do not deeply understand these topics and have

Permission to make digital or hard copies of all or part of this work for personal or classroom use is granted without fee provided that copies are not made or distributed for profit or commercial advantage and that copies bear this notice and the full citation on the first page. Copyrights for components of this work owned by others than ACM must be honored. Abstracting with credit is permitted. To copy otherwise, or republish, to post on servers or to redistribute to lists, requires prior specific permission and/or a fee. Request permissions from permissions@acm.org.

ITiCSE '19, July 15-17, 2019, Aberdeen, Scotland UK

(c) 2019 Association for Computing Machinery.

ACM ISBN 978-1-4503-6301-3/19/07 .\$15.00

https://doi.org/10.1145/3304221.3319760 little understanding of the serious security vulnerabilities that can be introduced by improper use. Although it has not been updated recently, integer overflow appeared in the most recent version (2011) of the twenty five most critical software vulnerabilities identified by the Common Weakness Enumeration [2], created by Mitre and the SANS Institute.

Visualization is an educational technique that is widely used to improve learning. We have developed a tool that uses visualization to help students understand integer security. The tool is part of a larger system called the Visualization and Analysis for C Code Security (VACCS) System. The VACCS system is designed to help students learn how to code securely in C. The Integer Representation (IR) component focuses on helping students to understand integer security. The IR window depicts the representation of integer types and the effects of wraparound and conversion from one integer type to another.

There are a number of pedagogical tools for visualization of security concepts, including network protocols and defenses $[4,9$, $16,18]$, formal models $[5,8,15]$ and cryptography [3, 6, 7, 11-13]. Benham proposed a method to teach data representation using a modular clock [1]. There are also visualization systems aimed to teach computer architecture $[14,17]$. Our tool focuses only on integer representation within the larger context of $C$ program security.

This paper describes our experience with teaching integer security within a required junior-level course on systems programming in $\mathrm{C}$ at Michigan Technological University. Section 2 describes our experience teaching the subject matter. Section 3 describes the visualization tool. Section 4 describes our use and evaluation of the tool. Our conclusions are given in section 5 . The evaluation results indicate that students felt the tool was useful and that students' knowledge of the material was improved during the course.

\section{TEACHING INTEGER SECURITY}

\subsection{Motivation}

Naive and sloppy use of integers can introduce subtle and significant security vulnerabilities into a $\mathrm{C}$ program. Consider the code in figure 1 [10]. (Line numbers have been added as a convenience.).

A user inputs the size of some data and the data itself as arguments one and two to the program. The program then makes a copy of the buffer.

This provides a useful motivating example when beginning a discussion on integer security. It represents code that a student is likely to write in an attempt to prevent writing beyond the bounds of a statically allocated buffer, in this program the buffer buf.

The code is indicative of a common failure by students to consider the entire range of values for a signed integer. For example, they 


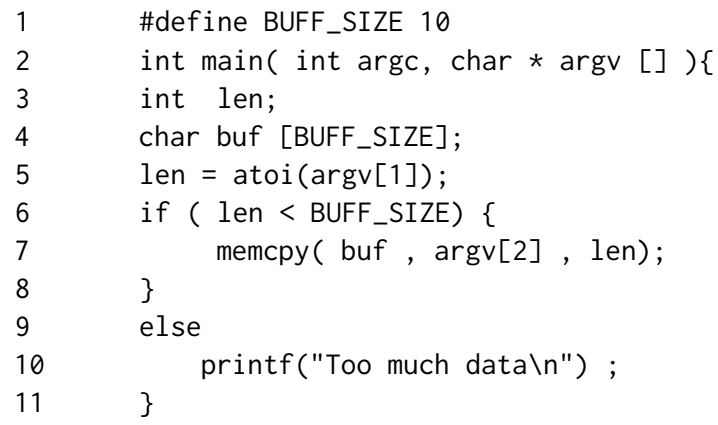

Figure 1: C Code to Copy a User Input Buffer

commonly consider only positive values where they expect positive values. If the value of len, set at line 5 , is negative, the code at line 7 will be executed.

The code at line 7 exposes that a student may be sloppy about noting type conversions that take place on a procedure call; and may misunderstand the effect of converting between signed and unsigned types. The third argument to memcpy is type size_t (unsigned) so that the amount of data copied can far exceed the 10 bytes allocated to buf. This results in a classic buffer overflow in which memory beyond buf can be overwritten.

In order to avoid these errors, students need to understand how integers are represented, when conversions take place within a $\mathrm{C}$ program, how conversions are performed, and the resulting values. These topics are commonly covered in undergraduate courses in $\mathrm{C}$ and computer organization.

\subsection{Curriculum}

In order to integrate important security concepts throughout our undergraduate curriculum, we decided to cover integer security within a junior-level systems programming course. This course teaches students about processes, linking and loading, I/O, IPC and network programming through the LINUX system call interface. This course also covers buffer overflows and stack protections. Students are required to code in $\mathrm{C}$. The course prerequisites are a course in $\mathrm{C}$ programming for Java programmers and a course in computer organization. Our curriculum had previously included the material on integer security and buffer overflows in a senior-level elective on computer security.

On the first day of class, we gave a short quiz to identify student understanding of the use of integers within a $\mathrm{C}$ program. There were four questions in total with one point per question. The quiz questions are given in table 1 . Question 1 asked students to identify the effects of conversion from a larger signed type to a smaller signed type. It required that students recognize the binary representation of the minimum representable value for a signed integer and that this binary value would be truncated in order to perform the conversion. ${ }^{1}$ Question 2 asked students to identify the effects of an integer overflow. Question 3 required students to understand the binary representation of the smallest representable unsigned type, the truncation on conversion, and the corresponding signed value. Question 4 required students to understand the representation of

\footnotetext{
${ }^{1}$ Students were given the size in bytes of all types used in the quiz.
}

-2 , identify the conversions applied (one at the division, resulting in an unsigned, and one at the assignment) and determine the effects of these conversions.

Table 1: Pre-test Questions

\begin{tabular}{|c|c|}
\hline Q1 & $\begin{array}{l}\text { short int } \mathrm{x} \text {; } \\
\text { int } \mathrm{i} \text {; } \\
\mathrm{i}=\mathrm{INT} \text { ININ; } \\
\mathrm{x}=\mathrm{i} \text {; } \\
\text { What is the value of } x ?\end{array}$ \\
\hline Q2 & $\begin{array}{l}\text { int } \mathrm{x} ; \\
\mathrm{x}=\mathrm{INT} \text { _MAX; } \\
\mathrm{x}=\mathrm{x}+3 \text {; } \\
\text { What is the value of } x ?\end{array}$ \\
\hline Q3 & $\begin{array}{l}\text { short int } \mathrm{x} \text {; } \\
\text { unsigned int ui; } \\
\text { ui=UINT_MAX; } \\
\mathrm{x}=\text { ui; } \\
\text { What is the value of } x ?\end{array}$ \\
\hline $\mathrm{Q} 4$ & $\begin{array}{l}\text { signed } \mathrm{s} \\
\text { unsigned } \mathrm{u} \\
\text { signed } \mathrm{x} \\
\mathrm{s}=-2 ; \mathrm{u}=2 \\
\mathrm{x}=\mathrm{s} / \mathrm{u} \\
\text { What is the value of } x ?\end{array}$ \\
\hline
\end{tabular}

Student performance is given in table 2. The mean represents the average score received on the one point question. It also shows the percentage of students who answered that question correctly as no partial credit was given.

Table 2: The Means $(\mu)$, Standard Deviations $(\sigma)$ of the Pretest Questions

\begin{tabular}{|l|l|l|l|l|}
\hline & Q1 & Q2 & Q3 & Q4 \\
\hline$\mu$ & 0.28 & 0.06 & 0.05 & 0.08 \\
\hline$\sigma$ & 0.45 & 0.24 & 0.21 & 0.27 \\
\hline
\end{tabular}

We were surprised at the class performance. Students performed poorly on all questions. This meant that our standard coverage was inadequate to root this material into the students' working knowledge. It also confirmed the utility of reviewing the material with an eye toward integer security.

The coverage roughly followed the integer security discussion in Secure Coding in C and C++, by Robert Seacord [10]. We reviewed integer representation, conversions, and operations (addition, subtraction, multiplication and division). Throughout the discussion, we included examples of security errors that result from improper use of integers.

\section{INTEGER REPRESENTATION WINDOW}

\subsection{Design}

The Visualization and Analysis for C Code Security (VACCS) system has several visualization components aimed at helping students to better understand how programs execute and coding practices that can create security vulnerabilities. A student runs an analysis program against her $\mathrm{C}$ code to produce a sequence of events. Each event corresponds to the execution of one source code line. These events are input to a visualization system which provides 
several perspectives on the execution. These perspectives include the Program Address Space (PAS), Call Graph (CG), Sensitive Data handling (SD) and File Operations (FO). Students step the visualization forward or backward through the events, or allow the system to execute the events without user intervention using a user-specified time interval between the events. This paper focuses on the Integer Representation (IR) component of the PAS perspective.

The IR window was designed to help students understand the effect of conversion from one type to another. We focused on identifying the number of bytes for a given type, changes in the number of bytes and in bit values that occur on a particular conversion, and the associated decimal value of these bit patterns when two's complement representation is used. ${ }^{2}$ At the time the tool was designed, we felt that students would not have difficulty understanding when conversions would be applied and the resulting types.

\subsection{Use}

The VACCS system comes up on the PAS tab, as shown in figure 2. There are tabs for each of the visualizations as well as the source code. Any of these windows can be detached from the main window to be a standalone window for the convenience of side-by-side reference. In this depiction, the source code has already been detached. The source code window is shown in figure 3 . The next line to be executed is highlighted in yellow.

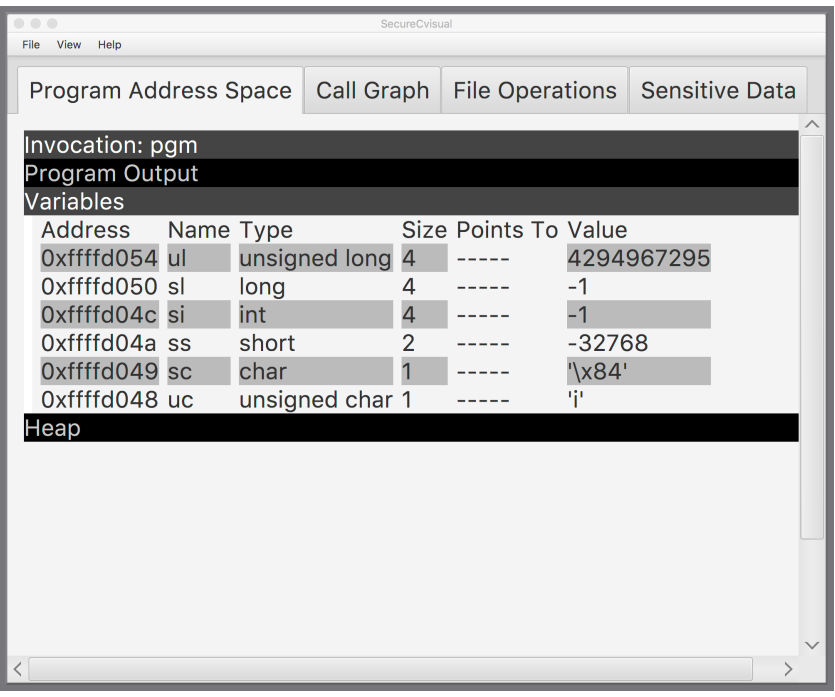

Figure 2: VACCS Program Address Space Visualization

\footnotetext{
${ }^{2}$ Two's complement was included in both of our lecture and tool as it is the most common method of representing signed integers on computers. Ones complement was also included in our lecture, but was not incorporated in the tool due to its less common use.
}

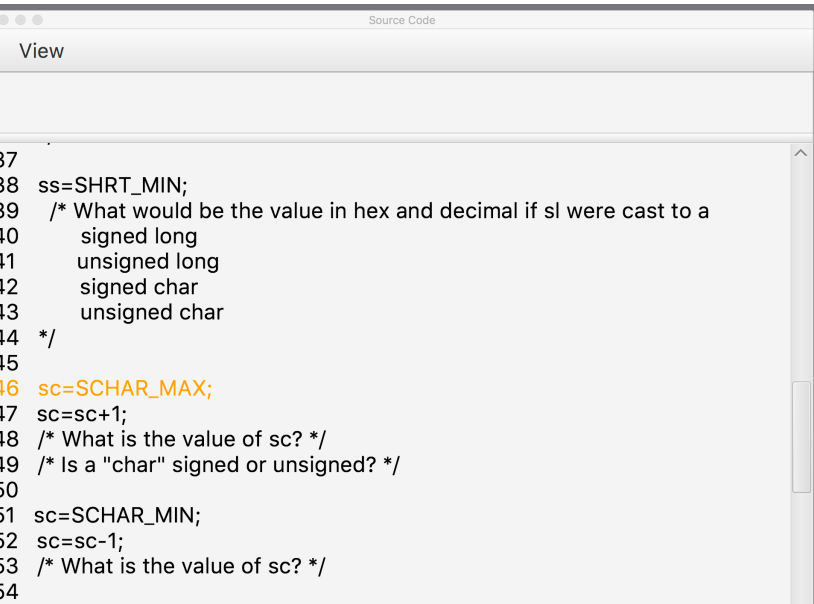

Figure 3: VACCS Source Code Window

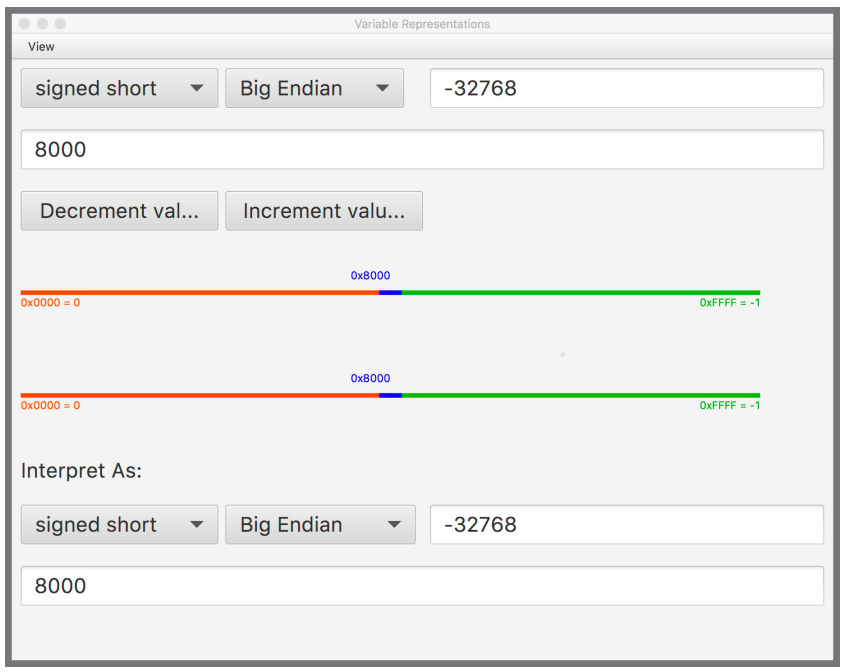

Figure 4: VACCS Integer Representation Window

A student brings up the IR window by clicking on the value of an integer variable in the PAS. Figure 4 shows the IR window that results from clicking on the value of the signed short integer variable ss from the main window depicted in figure 2 . The value of ss has just been set to SHRT_MIN which is -32768 .

The window has several features that allow a student to explore representation and conversion of the selected value. There are two representations of the chosen number, one at the top of the window and one at the bottom. Both the hexadecimal value for the corresponding bytes and the decimal value represented ( 8000 and -32768 , respectively, in figure 4) are shown. This allows a student to modify the parameters of the depicted value and look at the relevant values before and after a conversion. The endianess is also shown. Associated with each representation is a horizontal line scaled by the range of values (in hexadecimal and decimal) that can be represented by the given type. Negative values are shown in red, positive values are shown in green, and the current value is shown in blue. 
The byte order defaults to big endian. Selection of little endian from the drop down menu depicts the value that results from interpreting the same bit pattern in little endian order. Figure 5 shows the IR window of figure 4 after Little Endian is selected.

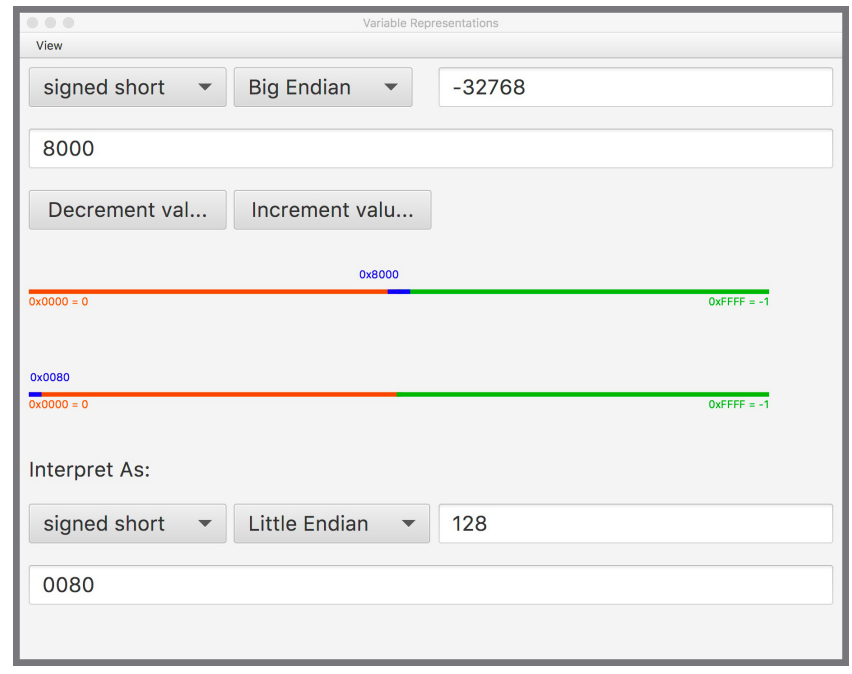

Figure 5: Big Endian and Little Endian Representations

The student can cast the value to another type by choosing a different type from the drop down menu in the representation given in the bottom half of the window. Figure 6 shows the signed short value cast to a signed int.

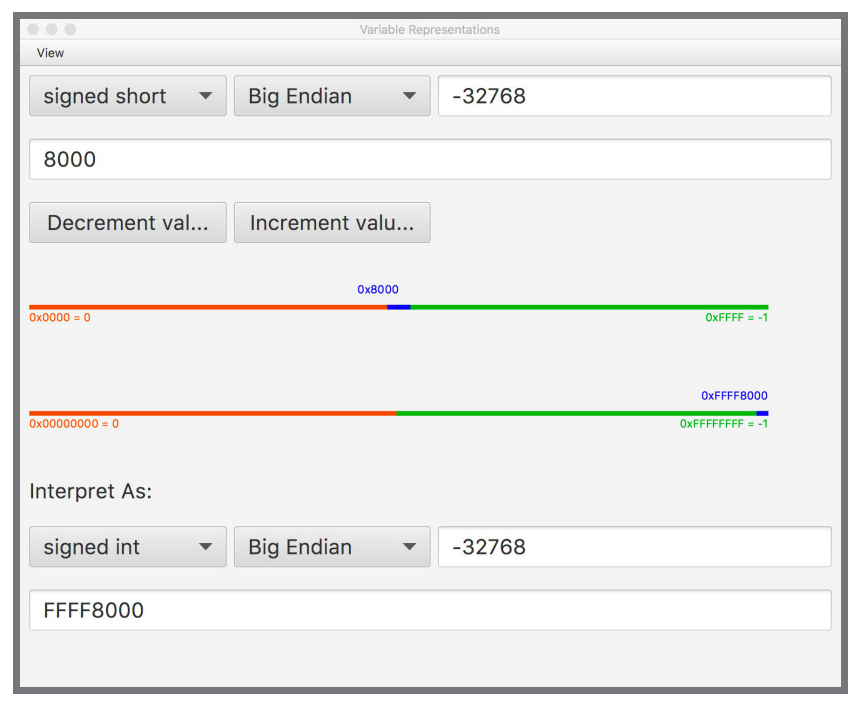

Figure 6: Signed Short and Signed Int Representations

A student can modify the depicted value in two ways. She can edit the decimal value in the upper representation or she can use the increment and decrement value buttons. All these actions operate against both the upper and lower representations. Figure 7 shows the IR window after the value shown in figure 4 is decremented.

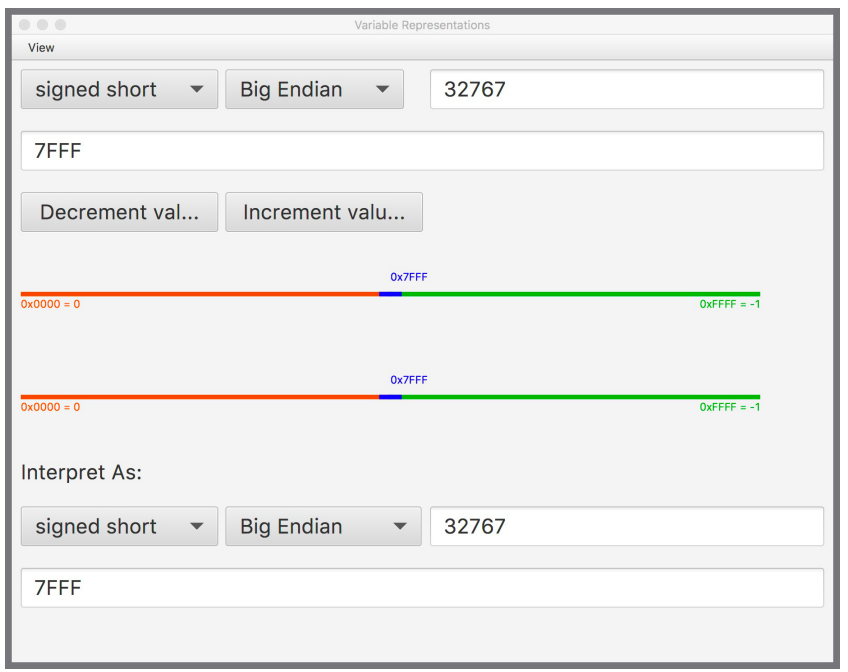

Figure 7: Decrement of SHRT_MIN

\section{EVALUATION}

\subsection{Environment, Procedure and Goals}

The tool was evaluated at Michigan Technological University in a required junior-level systems programming course with a total of 65 students. Students were given a pre-test on their knowledge prior to any lecture over the material.

Lectures were then given on integer representation, overflow, how and when integer type conversions are performed, common errors and methods to avoid these errors. The IR window was used during the lecture to demonstrate the mechanics of conversions between integer types: the number of bytes before and after the conversion, the bit values, and the corresponding decimal values when the representation is in two's complement. These in-class examples were similar to the questions that appeared on a post-test. Students were then given an extra credit assignment in which they used the IR window of the VACCS system to answer questions over the lecture material. After the extra credit assignment, students evaluated their experience with using the IR window. The knowledge of all students was assessed through questions on a post-test in the form of a midterm exam. We collected 59 valid pre-tests, 60 valid post-tests, and 25 valid evaluation forms.

The pre-test and post-test used essentially the same set of questions. These questions were discussed in section 2.2 and are given in table 1 . The IR window was used to present all the knowledge required to answer questions Q1, Q2, and Q3. Question Q4 not only required a type conversion, like the other questions, but required that students identify when a conversion must be performed and the resulting type. We did not included this in design of the IR window, or spend much time on it during lecture, because we believed students would not have difficulty with this material.

\subsection{Test Performance}

Table 3 and figure 8 have the mean, standard deviation and confidence intervals $\left(\mathrm{CI}^{-}\right.$and $\left.\mathrm{CI}^{+}\right)$of each question in the pre-test and post-test. The correctness of all questions improved in the post-test. 
Q1 and Q2 showed a $51 \%$ and $41 \%$ increase in correctness, respectively. Q3 showed a 72\% improvement, jumping from the lowest mean in the pre-test to the second highest mean in the post-test. Q4 only improved by $2 \%$, and has a correctness of $10 \%$ in the post-test.

Table 3: The Means $(\mu)$, Standard Deviations $(\sigma)$ and $95 \%$ Confidence Intervals of the Pre- and Post-test Questions

\begin{tabular}{|c|c|c|c|c|}
\hline \multicolumn{5}{|c|}{ Pre-test } \\
\hline & Q1 & Q2 & Q3 & Q4 \\
\hline$\mu$ & 0.28 & 0.06 & 0.05 & 0.08 \\
\hline$\sigma$ & 0.45 & 0.24 & 0.21 & 0.27 \\
\hline$C I^{-}$ & 0.17 & 0.00 & 0.00 & 0.01 \\
\hline$C I^{+}$ & 0.39 & 0.12 & 0.10 & 0.14 \\
\hline \multicolumn{5}{|c|}{ Post-test } \\
\hline & Q1 & Q2 & Q3 & $\mathrm{Q} 4$ \\
\hline$\mu$ & 0.79 & 0.47 & 0.77 & 0.10 \\
\hline$\sigma$ & 0.41 & 0.50 & 0.42 & 0.30 \\
\hline$C I^{-}$ & 0.69 & 0.34 & 0.67 & 0.00 \\
\hline$C I^{+}$ & 0.89 & 0.59 & 0.88 & 0.17 \\
\hline
\end{tabular}

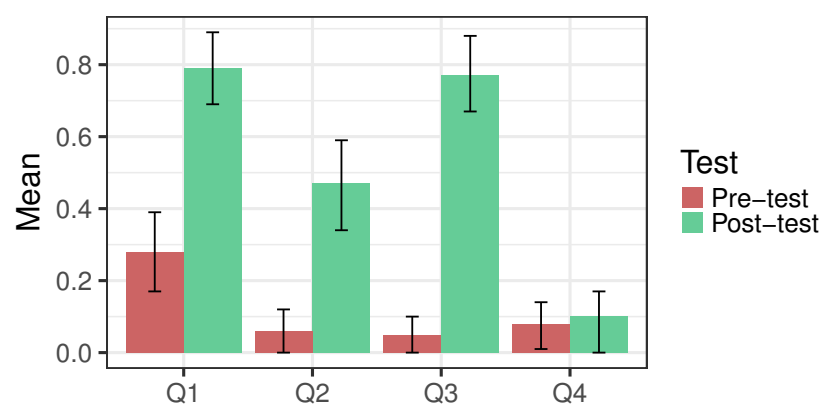

Figure 8: The Means with 95\% Confidence Intervals of the Pre- and Post-test Questions

We further used ANOVA (parametric) to investigate whether students' performance throughout the tests had significantly improved and employed the Kruskal-Wallis (KW) test (non-parametric) for confirmation of the result, both at $95 \%$ significant level. The $p$-values from both tests had values less than 0.05 in Q1, Q2, and Q3. With their increased means from the pre-test to the post-test (table 3), this indicates that the performance on integer representation and type conversion significantly improved. Hence, students showed significant improvement in Q1, Q2 and Q3 in the post-test.

Students did not show significant improvement in Q4. We expect this is because they could not give a correct answer unless they identified the conversion to unsigned at the division. (Most students answered "-1".) We had expected that students would be able to identify when conversions take place within a C program, so we did not add it to the visualization system or spend a significant amount of lecture time on this material.

Based on the response to Q4, we believe this material merits greater attention. In order to facilitate instruction and self-learning, we are developing a new visualization to help students identify when conversions take place. This visualization will take a single equation as input. It will depict the abstract syntax tree for the equation and step through each component operation. The visualization will show operand types for each operation and the type that results from applying the rules for integer conversions. The rules for conversion will be displayed and the rule applied at each operation will be identified. This course does not require homework, so students may also need more practice with this material.

\subsection{Subjective Evaluation}

We used a set of questions (table 4) to collect information on student perception of the effectiveness of the tool. We also gathered information on the time spent using the IR window. The first 10 rating questions evaluated the effectiveness of the IR window. The choices were: 1:strongly disagree, 2:disagree, 3:neutral, 4:agree, and 5:strongly agree. Q11 to Q13 evaluated the time participants spent on the tool. The choices for Q11 were 1:less than 5 mins, 2:5-9 mins, 3:10-14 mins, 4:15-29 mins, and 5:more than 30 mins. The choices for Q12 are 1:once, 2:twice, 3:3-4 times, 4:5-10 times, and 5:more than 10 times. The choices for Q13 were 1:less than 5 mins, 2:5-14 mins, 3:15-29 mins, 4:30-60 mins, and 5:more than 1 hour.

Table 4: IR Window Rating and Usage Questions

\begin{tabular}{|c|c|}
\hline \multicolumn{2}{|r|}{ Rating Questions } \\
\hline Q1 & $\begin{array}{l}\text { The IR window helped me to see how integer type conver- } \\
\text { sions are performed }\end{array}$ \\
\hline Q2 & $\begin{array}{l}\text { I understand integer type conversions better after using the } \\
\text { IR window }\end{array}$ \\
\hline Q3 & $\begin{array}{l}\text { By using the IR window, I was able to identify concepts I did } \\
\text { not understand }\end{array}$ \\
\hline Q4 & $\begin{array}{l}\text { The IR window enhanced the course in the area of integer } \\
\text { type conversion }\end{array}$ \\
\hline Q5 & The text of the IR window was easy to read \\
\hline Q6 & The interface for integer conversion was intuitive \\
\hline Q7 & $\begin{array}{l}\text { The depiction of the value range for (un)signed integers was } \\
\text { easy to read }\end{array}$ \\
\hline Q8 & $\begin{array}{l}\text { The depiction of the value range of (un)signed integers was } \\
\text { easy to understand }\end{array}$ \\
\hline Q9 & $\begin{array}{l}\text { The depiction of value range of (un)signed integers helped } \\
\text { me to understand integer type conversions }\end{array}$ \\
\hline Q10 & $\begin{array}{l}\text { The Increment and Decrement functions were useful for ex- } \\
\text { ploring incrementing/decrementing an integer beyond its } \\
\text { max or min }\end{array}$ \\
\hline \multicolumn{2}{|r|}{ Usage Questions } \\
\hline Q11 & $\begin{array}{l}\text { How long did it take to understand integer conversion using } \\
\text { the tool }\end{array}$ \\
\hline Q12 & How many times did you use the software \\
\hline Q13 & How long did you use the software in total \\
\hline
\end{tabular}

Table 5 lists the mean, standard deviation and confidence intervals for each of the evaluation questions. Figure 9 depicts the means and confidence intervals for both the evaluation and usage questions.

The rating of the tool was generally positive with a mean of 4.00 . Q10 received the highest rating of 4.6, which means that students believed that the Increment and Decrement functions were useful for exploring the effects of incrementing and decrementing an integer beyond its maximum and minimum. Q7, which is associated 
with the readability of the depiction of the range of values for (un)signed integers at the right of IR window, had the lowest rating of 3.56. Initially the system used a circular representation to depict the range of decimal values. This was changed to a line (as seen in the figures of section 3) in response to the student comments. The means of Q11, Q12 and Q13 are 2.52, 2.08 and 3.32. This indicates that students generally used the IR Window twice for 10 to 15 minutes each time and 15 to 30 minutes in total.

Table 5: The Means $(\mu)$, Standard Deviations $(\sigma)$, and Confidence Intervals of Evaluation Questions

\begin{tabular}{|c|c|c|c|c|c|}
\hline & Q1 & Q2 & Q3 & Q4 & Q5 \\
\hline$\mu$ & 4.48 & 4 & 3.82 & 4.32 & 3.96 \\
\hline$\sigma$ & 0.59 & 0.76 & 0.80 & 0.56 & 0.93 \\
\hline$C I^{-}$ & 4.25 & 3.70 & 3.51 & 4.10 & 3.59 \\
\hline$C I^{+}$ & 4.71 & 4.30 & 4.13 & 4.54 & 4.33 \\
\hline & Q6 & Q7 & Q8 & Q9 & Q10 \\
\hline$\mu$ & 4 & 3.56 & 3.64 & 3.64 & 4.6 \\
\hline$\sigma$ & 0.82 & 1.04 & 0.95 & 0.99 & 0.58 \\
\hline$C I^{-}$ & 3.68 & 3.15 & 3.27 & 3.25 & 4.37 \\
\hline$C I^{+}$ & 4.32 & 3.97 & 4.01 & 4.03 & 4.83 \\
\hline
\end{tabular}

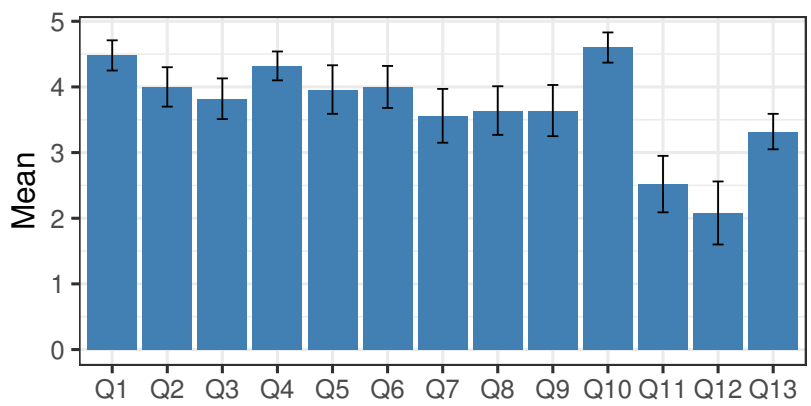

Figure 9: The Means with Confidence Intervals of Evaluation Rating and Usage Questions

The four write-in questions were used to collect information of the participants' major, their thoughts on the IR window, features to add, and problems with installation. Regarding the IR window, the feedback was generally positive in its functionality, layout and usage. Many students enjoyed the "nice layout" and stated that " $I$ like using the IR window. It made it easy to visualize when a value would roll over. It was good to be able to see when only the hex or decimal value changed and to think about why", "It was very helpful to have a visual that encompassed all the important aspects of int conversions and abstracted away from doing the math by hand" and "It is very good for showing integer type conversions. I have not seen an easier to use program that shows such important information in a GUI so perfectly designed for someone to pick up and use as they please".

Most students did not encounter problems with the installation. A few students had an issue running Java UISplashScreen (the current tool name) on Windows machines. This could have been a Java version problem, as one student suggested. When asked to suggest features to add, the majority of students found the available features satisfying. There were also some comments for further improvements. A student suggested "showing how mathematical operators changed a number during its process that would be great". Some students found that "the circle on the right side of IR window is not easy to interpret" and suggested that "some descriptions should be added". We felt this was partially addressed when we changed the range depiction from a circle to a line. We are considering addition of a help button.

\section{CONCLUSIONS AND FUTURE WORK}

The lecture together with use of the IR window to present examples during class significantly increased students' understanding of the material as measured through a quiz. Additionally, students felt like the IR window enhanced the course. We are extending the IR window to allow students to predict or view the type changes that occur during the evaluation of a $\mathrm{C}$ language statement. The additions will include the ability to visualize the entire type coercion process and how intermediate values are affected by $\mathrm{C}$ semantics. Students will be able to step through the computations and type coercions that the compiler must implement to generate valid code.

The post-test results and evaluation were encouraging for use of the tool to improve learning. Yet many students continued to find the material difficult. We plan to create a stand-alone tool that can be easily used in earlier courses or by students that are studying the material independently. This tool will include the features of the current IR window, add the type coercion enhancement described above, and will also present a binary representation of variable values including association of bytes with increasing hardware address.

The tool was used both during lecture and to provide (extra credit) homework problems for students. The VACCS system significantly enhanced the lectures. It proved to make lecture far more convenient by providing a quick, error-free mechanism to tie the representation of an integer variable with its decimal value. It was also convenient to discuss the conversion between any integer types by simply selecting the new type in the IR window. When students asked the inevitable "what if" questions, it was quick and straightforward to both explain and then demonstrate.

The most recent version of the VACCS system is available at: http://pages.mtu.edu/ jwwalker/vaccs.html.

\section{ACKNOWLEDGMENTS}

This work was supported in part by the National Science Foundation under grants DGE-1523017 and DUE-1245310.

\section{REFERENCES}

[1] James W. Benham. 1992. A Geometric Approach to Presenting Computer Representations of Integers. SIGCSE Bull. 24, 4 (Dec. 1992), 27-28.

[2] Steve Christey (Ed.). 2011. Common Weakness Enumeration. http://cwe.mitre. org/top25/ (Accessed: 2018-08-31).

[3] Derek Ebeling and Rob Santos. 2007. Public Key Infrastructure Visualization. F. Comput. Sci. Coll. 23, 1 (Oct. 2007), 247-254.

[4] Niakam Kazemi and Shiva Azadegan. 2010. IPsecLite: A Tool for Teaching Security Concepts. In Proceedings of the 41st ACM Technical Symposium on Computer Science Education (SIGCSE '10). ACM, New York, NY, USA, 138-142.

[5] Yifei Li, Steve Carr, Jean Mayo, Ching-Kuang Shene, and Chaoli Wang. 2012. DTEvisual: A Visualization System for Teaching Access Control Using Domain 
Type Enforcement. Fournal of Computing Science in College 28, 1 (October 2012), 125-132.

[6] Jun Ma, Jun Tao, Melissa Keranen, Jean Mayo, Ching-Kuang Shene, and Chaoli Wang. 2014. SHAvisual: A Secure Hash Algorithm Visualization Tool. In Proceedings of the 2014 conference on Innovation \& technology in computer science education. ACM, 338-338.

[7] Dino Schweitzer and Leemon C. Baird III. 2006. The design and use of interactive visualization applets for teaching ciphers. In Proceedings of the 7th Annual IEEE Information Assurance Workshop. 69-75.

[8] Dino Schweitzer, Mike Collins, and Leemon C Baird III. 2007. A visual approach to teaching formal models in security. In Proceedings of the 11th Colloquium for Information Systems Security Education (CISSE). 69-75.

[9] Dino L. Schweitzer, Leemon C. Baird III, Mike D. Collins, Wayne C. Brown, and Mike Sherman. 2006. GRASP: A visualization tool for teaching security protocols In Proceedings of the 10th Colloquium for Information Systems Security Education. 75-81.

[10] Robert C. Seacord. 2013. Secure Coding in C and C++ (2nd ed.). Addison-Wesley Professional.

[11] Jun Tao, Jun Ma, Melissa Keranan, Jean Mayo, and Ching-Kuang Shene. 2012. ECvisual: A Visualization Tool for Elliptic Curve Based Ciphers. In roceedings of the 43rd ACM technical symposium on Computer Science Education. ACM, 571-576.
[12] Jun Tao, Jun Ma, Melissa Keranen, Jean Mayo, and Ching-Kuang Shene. 2011. DESvisual: A Visualization Tool for the DES Cipher. Fournal of Computing Science in College 27, 1 (October 2011), 81-89.

[13] Jun Tao, Jun Ma, Melissa Keranen, Jean Mayo, Ching-Kuang Shene, and Chaoli Wang. 2014. RSAvisual: A Visualization Tool for the RSA Cipher. In Proceedings of the 45th ACM technical symposium on Computer science education. ACM, 635-640.

[14] Kenneth Vollmar and Pete Sanderson. 2006. MARS: An Education-oriented MIPS Assembly Language Simulator. In Proceedings of the 37th SIGCSE Technical Symposium on Computer Science Education (SIGCSE '06). ACM, 239-243.

[15] Man Wang, Steve Carr, Jean Mayo, Ching-Kuang Shene, and Chaoli Wang. 2014. MLSvisual: A Visualization Tool for Teaching Access Control Using Multi-Level Security. In Proceedings of the 2014 conference on Innovation \& technology in computer science education. ACM, 93-98.

[16] Justin Warner, David Musielewicz, G. Parks Masters, Taylor Verett, Robert Winchester, and Steven Fulton. 2010. Network Firewall Visualization in the Classroom. f. Comput. Sci. Coll. 26, 2 (Dec. 2010), 88-96.

[17] Cecile Yehezkel, Mordechai Ben-Ari, and Tommy Dreyfus. 2005. Computer Architecture and Mental Models. In Proceedings of the 36th SIGCSE Technical Symposium on Computer Science Education (SIGCSE '05). ACM, 101-105.

[18] Xiaohong Yuan, Percy Vega, Yaseen Qadah, Ricky Archer, Huiming Yu, and Jinsheng Xu. 2010. Visualization Tools for Teaching Computer Security. Trans. Comput. Educ. 9, 4, Article 20 (Jan. 2010), 28 pages. 